\title{
Chemiluminescence of carbon dots under strong alkaline solutions: a novel insight into carbon dot optical propertiest
}

Cite this: Nanoscale, 2013, 5, 2655

Received 21st January 2013

Accepted 8th February 2013

DOI: 10.1039/c3nr00358b

www.rsc.org/nanoscale

\author{
Lixia Zhao, ${ }^{*}$ Fan Di, Dabin Wang, Liang-Hong Guo, ${ }^{*}$ Yu Yang, Bin Wan and Hui Zhang
}

We report the surprising chemiluminescence $(\mathrm{CL})$ behavior of fluorescent carbon dots in the presence of a strong alkaline solution, such as $\mathrm{NaOH}$ or $\mathrm{KOH}$. The $\mathrm{CL}$ intensity was dependent on the concentration of the base and carbon dots in a certain range. A possible $\mathrm{CL}$ mechanism was studied by UV-Vis, fluorescence, CL, FTIR, XPS and EPR spectroscopy. Radiative recombination of the injected electrons by "chemical reduction" of carbon dots with thermally excited generated holes was proposed, which sheds new light on the characteristics of carbon dots.

As a rising star of the nanocarbon family, carbon nanodots have attracted increasing attention due to their excellent luminescent properties and wide applications in many areas of fundamental and technical importance. ${ }^{1}$ Intense research still focuses on their further application and intrinsic luminescence mechanism.

Luminescent properties of carbon dots are usually investigated by photoluminescence (PL) produced using photoexcitation, ${ }^{2-8}$ and electrochemiluminescence (ECL) generated by electron injection..$^{9-12}$ However, chemiluminescence (CL) generated from chemical energy excitation through a chemical reaction has been rarely used to study the optical properties of carbon dots. To the best of our knowledge, only $\operatorname{Lin}^{13-15}$ and Cui's ${ }^{\mathbf{1 6}}$ groups have recently described the CL behavior of carbon dots when they coexist with oxidants $\left(\mathrm{KMnO}_{4}, \mathrm{Ce}(\mathrm{IV})\right.$, $\left.\mathrm{NaIO}_{4}\right)$ or an ultraweak chemiluminescence system $\left(\mathrm{H}_{2} \mathrm{O}_{2}-\right.$ $\mathrm{NaHSO}_{3}, \mathrm{H}_{2} \mathrm{O}_{2}-\mathrm{HNO}_{2}$ ) under acidic conditions. Herein, we observed for the first time that CL was generated when only a strongly alkaline solution was injected into the carbon dots, without the presence of any CL reagent, CL system or oxidants. Our initial purpose was to study the CL behavior of carbon dots

State Key Laboratory of Environmental Chemistry and Ecotoxicology, Research Center for Eco-Environmental Sciences, Chinese Academy of Sciences, P.O. Box 2871, Beijing 100085, P.R. China. E-mail: zlx@rcees.ac.cn; lhguo@rcees.ac.cn; Fax: +86-1062849685; Tel: $+86-10-62849338$

† Electronic supplementary information (ESI) available. See DOI: $10.1039 / \mathrm{c} 3 \mathrm{nr} 00358 \mathrm{~b}$ using an alkaline solution as a reaction medium to improve the CL intensity. Surprisingly, the addition of high concentration $\mathrm{NaOH}$ solution to the carbon dots was accompanied by a fast CL phenomenon. This investigation provided new insight into the physical-chemical and optical properties of carbon dots.

The carbon dots with a diameter of $2-4 \mathrm{~nm}$ (Fig. $\mathrm{S} 1$ in the ESI $\dagger$ ) were synthesized by the microwave treatment described in the literature with a slight modification (see ESI $\dagger$ ), using glucose as the carbon source and PEG 1500 as the co-reactant. ${ }^{9}$ As with the other carbon dots, the synthesized nanomaterial exhibited a UV-Vis absorption band at $280 \mathrm{~nm}$, attributed to the $\mathrm{n}-\pi^{*}$ transition of the $\mathrm{C}=\mathrm{O}$ band and a $\pi-\pi^{*}$ transition of the conjugated $\mathrm{C}=\mathrm{C}$ band. ${ }^{17}$ The fluorescence (FL) emission shifted as the excitation wavelength increased. When the emission reached its maximum, the peak centered at $525 \mathrm{~nm}$. Interestingly, with the addition of $\mathrm{NaOH}$ into the carbon solution, the colour of the carbon dot aqueous solution darkened from light yellow to yellow-brown. A new absorption peak at $272 \mathrm{~nm}$ appeared in the UV-Vis spectrum, but no appreciable absorption peak was observed under $280 \mathrm{~nm}$ for the original carbon

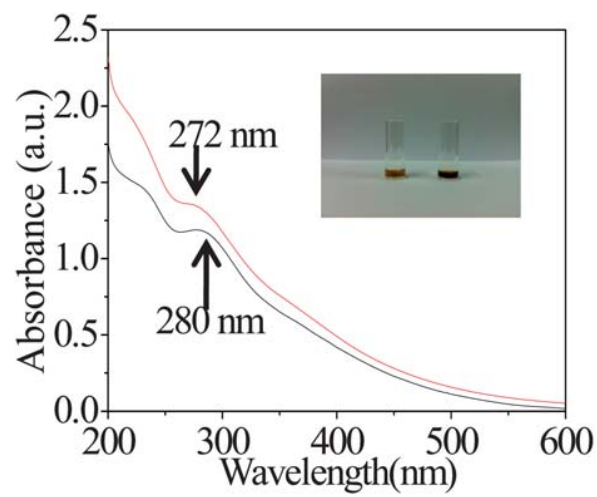

Fig. 1 The UV-Vis absorption spectra of pristine carbon dots and $\mathrm{NaOH}$-treated carbon dots. The inset is the photograph of the carbon dots only (left) and carbon dots in $\mathrm{NaOH}$ solution (right), in which the carbon dots are in the dilution of $1: 1600$, and $\mathrm{NaOH}$ concentration is $2 \mathrm{M}$. 
dots (Fig. 1). Changes in the surface structure of the carbon dots were thought to be the cause of the new absorption feature. ${ }^{18}$ With addition of $\mathrm{NaOH}$, the maximum FL emission intensity decreased obviously, while a slight red shift of the maximum emission wavelength was observed (Fig. S2 in the ESI $\dagger$ ). Meanwhile, a fast chemiluminescence signal appeared (Fig. 2). The $\mathrm{CL}$ intensity was dependent on the concentration of $\mathrm{NaOH}$ and carbon dots (Fig. 2A-C) in a certain range. A similar phenomenon was also observed with the addition of high concentration $\mathrm{KOH}$ (Fig. S3A in the ESI $\dagger$ ). The above results also suggest that the carbon dots have potential as FL or CL sensors for high $\mathrm{pH}$ measurement.

To validate the universal chemiluminescent nature of the carbon dots in the presence of a strong alkaline solution, carbon dots were prepared by pyrolysis of ethylenediaminetetraacetic acid (EDTA) salts, ${ }^{19}$ and similar CL behaviour was accompanied with the addition of different concentrations of $\mathrm{NaOH}$ (Fig. S3B in the ESI $\dagger$ ).

In order to verify the chemiluminescent characteristics of the carbon dots, CL spectra of the carbon dots- $\mathrm{NaOH}$ solution system were studied using high-energy cutoff filters of various wavelengths. As shown in Fig. 2D, CL emission was located in a wide range of 400-600 $\mathrm{nm}$, and the maximum was centered at $555 \mathrm{~nm}$. The spectral range was similar to the fluorescence emission wavelength of the carbon dots. Hence, it was reasonable that CL could be attributed to the various surface energy traps that existed on the carbon dots. The CL spectrum was redshifted in comparison to the most intense PL spectrum, which mainly occurred through excitation and emission within the
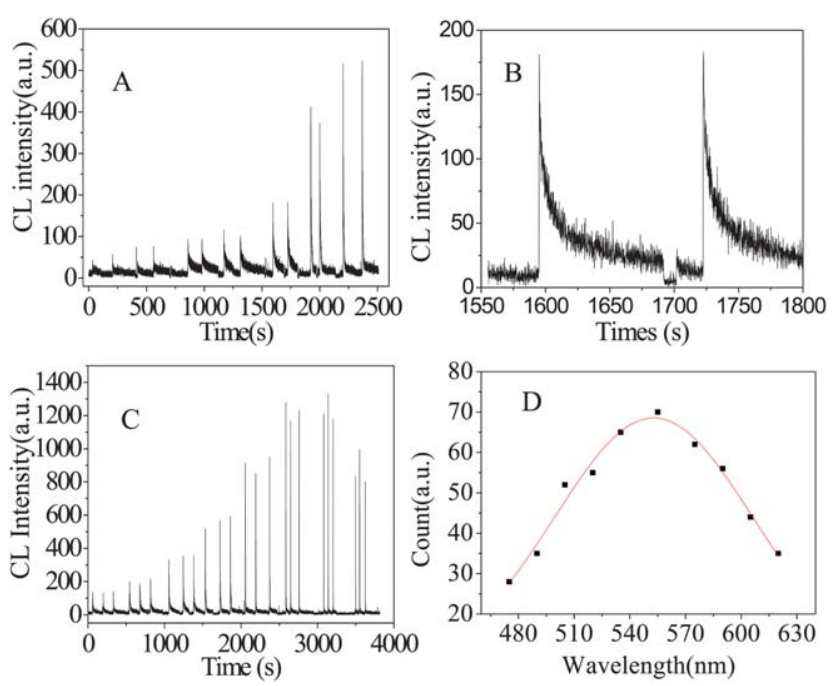

Fig. $2 C L$ kinetic curves $(A-C)$ and the $C L$ spectrum (D) of the carbon dot- $\mathrm{NaOH}$ system. (A) Carbon dots are in the dilution of $1: 32$, the concentration of $\mathrm{NaOH}$ is $0.05 \mathrm{M}, 0.1 \mathrm{M}, 0.5 \mathrm{M}, 1 \mathrm{M}, 2 \mathrm{M}, 5 \mathrm{M}$ and $8 \mathrm{M}$ respectively, from left to right. $\mathrm{CL}$ measurement at each $\mathrm{NaOH}$ concentration was carried out in duplicate. (B) The enlarged view of $(\mathrm{A})$ in which the carbon dots are in the dilution of $1: 32$, and the $\mathrm{NaOH}$ concentration is $2 \mathrm{M}$. (C) The concentration of $\mathrm{NaOH}$ is $2 \mathrm{M}$, the carbon dots are in the dilution of $1: 128,1: 64,1: 32,1: 16,1: 8,1: 4,1: 2$ and $1: 1 . \mathrm{CL}$ measurement for each carbon dot concentration was carried out in triplicate. (D) The $\mathrm{CL}$ spectrum of the carbon dot- $\mathrm{NaOH}$ system, in which the carbon dots are in the dilution of $1: 32$, and $\mathrm{NaOH}$ concentration is $2 \mathrm{M}$. core of the nanoparticles. The red shift most likely resulted from the smaller energy separations of the carbon dot surface states, compared with the energy for the most intense PL. ${ }^{20}$ Furthermore, superoxide dismutase (SOD), thiourea and histidine - the quenchers of the superoxide, hydroxyl radical and singlet oxygen - did not inhibit the CL signal. De-oxygenated solutions also had no inhibitory effect on the CL, which indicated that the dissolved oxygen had little effect on the CL, and the singlet oxygen CL emitter could be excluded in the carbon dots-NaOH system.

In order to find out what changes the carbon dots experienced when in the high concentration $\mathrm{NaOH}$ solution, pristine carbon dots and $\mathrm{NaOH}$-treated carbon dots were further characterized by Fourier-transformed infrared spectroscopy (FTIR) and X-ray photoelectron spectroscopy (XPS). The FTIR spectra (Fig. 3a) revealed that functional groups such as $\mathrm{C}-\mathrm{OH}$ (hydroxyl), $-\mathrm{CH}_{3},-\mathrm{CH}_{2}, \mathrm{C}-\mathrm{O}-\mathrm{C}$ (ether) and $\mathrm{C}=\mathrm{O}$ (carbonyl) formed on the carbon dots, in agreement with previous studies ${ }^{9}$ which used carbon dots prepared by microwave treatment methods. After the reaction, the $\mathrm{NaOH}$-treated carbon dots showed a significant reduction in the amount of carbonyl moieties and an increase in the amount of hydroxyl groups (Fig. 3b), which indicated that the surface carbonyl groups of the carbon dots were probably partly reduced to hydroxyl groups. From the XPS analysis (Fig. 4), it showed that pristine carbon dots were $70.64 \mathrm{wt} \%$ carbon and $29.36 \mathrm{wt} \%$ oxygen, whereas $\mathrm{NaOH}$-treated carbon dots were $76.47 \mathrm{wt} \%$ carbon and 23.37 wt $\%$ oxygen (Fig. 4A and B). An increase of hydrogen content and a decrease of oxygen content indicated hydrogenation and deoxygenation of carbon dots after reaction. Remarkably, the number of carbonyl groups decreased, and a C-O peak characteristic of hydroxyl groups increased at $\sim 286.6$ $\mathrm{eV}$. This is consistent with FTIR analysis. The $\mathrm{C}-\mathrm{H}$ peak intensity around $282.9 \mathrm{eV}$ also increased (C 1s peak, Fig. 4C).

A typical transmission electron microscope (TEM) image (Fig. S4A in the ESI $\dagger$ ) showed that the carbon dots aggregated in strong alkaline solutions, but $\mathrm{NaOH}$-treated carbon dots remained dispersed after dialysis (Fig. S4B in the ESI $\dagger$ ). We initially thought a Cannizzaro reaction might have happened

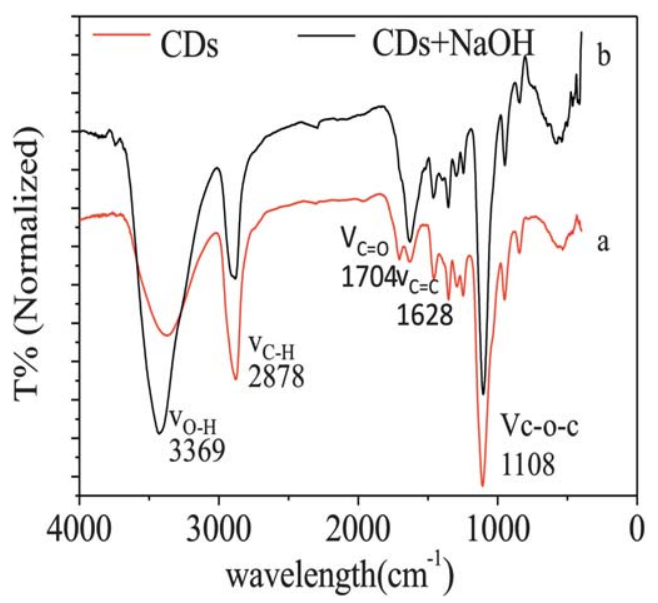

Fig. 3 FTIR spectra of pristine carbon dots (a) and $\mathrm{NaOH}$-treated carbon dots (b). 

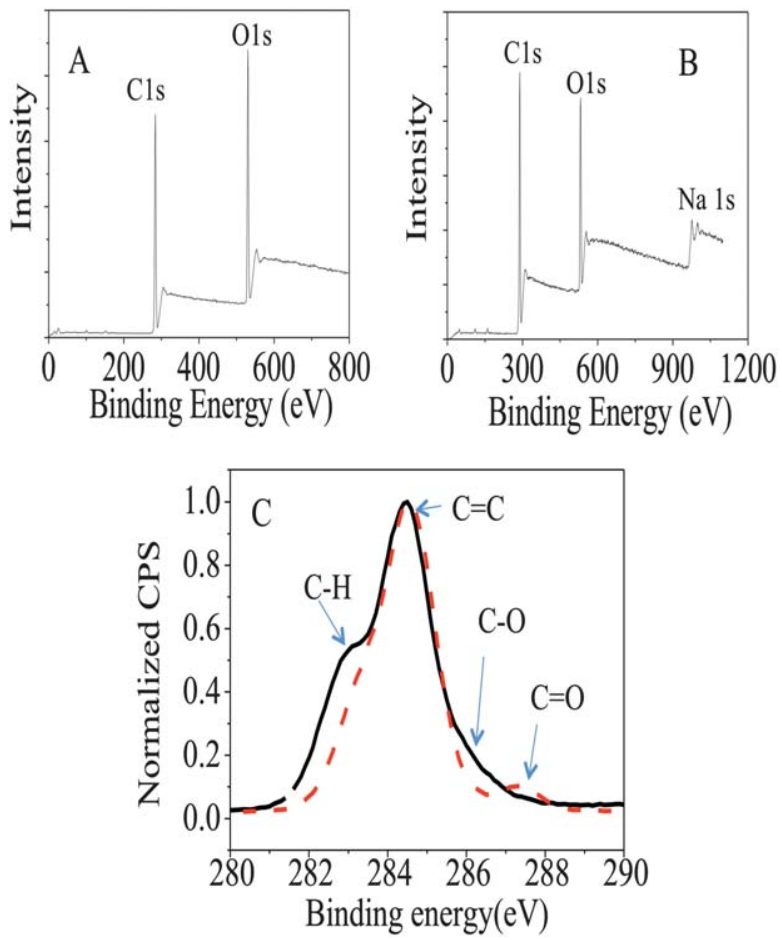

Fig. 4 XPS spectra of pristine carbon dots (A), NaOH-treated carbon dots (B), and the $C$ is peaks of carbon dots $(C)$ in which the dashed line is pristine carbon dots, and the solid line is $\mathrm{NaOH}$-treated carbon dots. The $\mathrm{Na}$ signal in (B) is attributed to residual $\mathrm{NaOH}$.

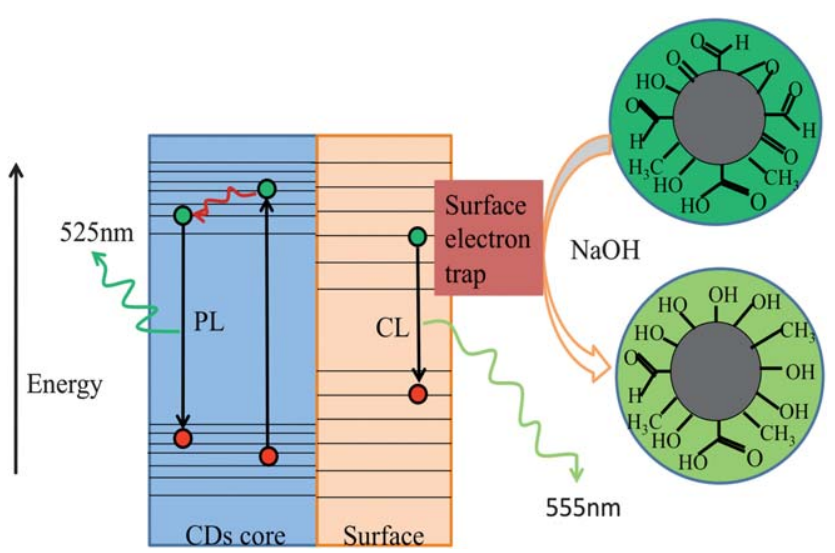

Fig. 5 Schematic illustration of the possible FL and $\mathrm{CL}$ mechanism of carbon dots in the high concentration $\mathrm{NaOH}$ system.

between the two carbon dots when the high concentration $\mathrm{NaOH}$ solution was added because aldehyde groups without $\alpha$ active hydrogen atoms are likely to occur on the surface of carbon dots according to FTIR analysis. However, typical Cannizzaro reactions usually involve relatively harsh reaction conditions with an excess of an alkaline metal hydroxide in an aqueous solution at high temperatures. In addition, FTIR and XPS analysis showed that there was no emergence of new vibrational absorption bands of carboxyl and carboxyl peaks at $289 \mathrm{eV}$ after the reaction. The results indicated that cannizzaro reactions had not happened.
Although the reaction mechanism remained unclear, deoxygenation of carbon dots under alkaline conditions appeared to be the reverse of the oxidation reaction of carbon dots in strong acids.,21 The mechanism was supported by the $\mathrm{NaOH}$ concentration dependency of this reaction - the higher the concentration of $\mathrm{NaOH}$ solution, the faster the reaction and also the higher the chemiluminescence intensity. This interesting reaction provided an explanation for the "chemical reduction" of the carbon dots as the deoxygenation of exfoliated GO. ${ }^{22}$

In order to further study the interesting CL phenomena, the ground-state luminescent species in the carbon dots were investigated and quantified through electron paramagnetic resonance (EPR). The carbon dots showed an EPR signal at 2.00094 (Fig. S5 of the ESI $\dagger$ ), which revealed a singly occupied orbital in the ground-state carbon dots. The singly occupied orbital indicated that carbon dots could be electron donors or acceptors during the reaction. EPR spectra of the carbon dots in a strong alkaline solution are also presented in Fig. S5. $†$ The $g$ value for the carbon dots increased from 2.00094 to 2.00218, which indicated a change in the singly occupied orbital in the carbon dots after the reaction, and an electron transfer reaction was suggested. In addition, the peak intensity and area both increased, which indicated that carbon dots were possible electron acceptors in the redox reaction under a strong alkaline solution.

Based on the above results, a possible mechanism of "chemical reduction" of the carbon dots in the high concentration $\mathrm{NaOH}$ solution could provide an explanation for the CL generation, as shown in Fig. 5. The single orbital detected by EPR could serve as an electron trap. The thermally excited generated holes can annihilate with the "chemical reduction" injected electrons to produce the CL. Few electrons and holes are available for the nanomaterials at absolute zero; as the temperature rises above the zero, there is more energy to excite the electron to the higher levels and more holes to be left. ${ }^{\mathbf{1 3 , 2 3}}$ The electron injection into carbon dots by the "chemical reduction" reaction increased the amount of electrons in the carbon dots and accelerated the electron-hole annihilation, which resulted in the energy release in the form of CL emission.

In conclusion, we found an interesting CL behavior of carbon dots in a strong alkaline solution. Possible CL mechanisms were studied by UV-Vis, FL, CL, FTIR, XPS and EPR. When the $\mathrm{NaOH}$ concentration was higher than $0.05 \mathrm{M}$, a fast CL signal appeared and the FL signal decreased. The CL and FL intensities were dependent on the concentration of the base. A possible CL mechanism of radiative recombination of the injected electrons by "chemical reduction" of the carbon dots and thermally excited generated holes was suggested to account for the surprising carbon dot CL behavior under strong alkaline conditions. This study provided new insight into the electron and hole state and the optical properties of carbon dots.

This work was supported by the Ministry of Science and Technology of China (2011CB936001 and 2010CB933502) and the National Natural Science Foundation of China (21177138, 20907060, 21077124, 21277158 and 21207146). 


\section{Notes and references}

1 J. C. G. E. da Silva and H. M. R. Goncalves, TrAC, Trends Anal. Chem., 2011, 30, 1327-1336.

2 Y. P. Sun, B. Zhou, Y. Lin, W. Wang, K. A. S. Fernando, P. Pathak, M. J. Meziani, B. A. Harruff, X. Wang, H. F. Wang, P. J. G. Luo, H. Yang, M. E. Kose, B. L. Chen, L. M. Veca and S. Y. Xie, J. Am. Chem. Soc., 2006, 128, 7756-7757.

3 S. L. Hu, K. Y. Niu, J. Sun, J. Yang, N. Q. Zhao and X. W. Du, J. Mater. Chem., 2009, 19, 484-488.

4 R. L. Liu, D. Q. Wu, S. H. Liu, K. Koynov, W. Knoll and Q. Li, Angew. Chem., Int. Ed., 2009, 48, 4598-4601.

5 X. J. Mao, H. Z. Zheng, Y. J. Long, J. Du, J. Y. Hao, L. L. Wang and D. B. Zhou, Spectrochim. Acta, Part A, 2010, 75, 553-557.

6 S. Chandra, S. H. Pathan, S. Mitra, B. H. Modha, A. Goswami and P. Pramanik, RSC Adv., 2012, 2, 3602-3606.

7 L. P. Lin, X. X. Wang, S. Q. Lin, L. H. Zhang, C. Q. Lin, Z. M. Li and J. M. Liu, Spectrochim. Acta, Part A, 2012, 95, 555-561.

8 Z. C. Yang, M. Wang, A. M. Yong, S. Y. Wong, X. H. Zhang, H. Tan, A. Y. Chang, X. Li and J. Wang, Chem. Commun., 2011, 47, 11615-11617.

9 H. Zhu, X. L. Wang, Y. L. Li, Z. J. Wang, F. Yang and X. R. Yang, Chem. Commun., 2009, 34, 5118-5120.

10 L. L. Li, J. Ji, R. Fei, C. Z. Wang, Q. Lu, J. R. Zhang, L. P. Jiang and J. J. Zhu, Adv. Funct. Mater., 2012, 22, 2971-2979.
11 L. Y. Zheng, Y. W. Chi, Y. Q. Dong, J. P. Lin and B. B. Wang, J. Am. Chem. Soc., 2009, 131, 4564-4565.

12 J. G. Zhou, C. Booker, R. Y. Li, X. L. Sun, T. K. Sham and Z. F. Ding, Chem. Phys. Lett., 2010, 493, 296-298.

13 Z. Lin, W. Xue, H. Chen and J. M. Lin, Chem. Commun., 2012, 48, 1051-1053.

14 W. Xue, Z. Lin, H. Chen, C. Lu and J. M. Lin, J. Phys. Chem. C, 2011, 115, 21707-21714.

15 Z. Lin, W. Xue, H. Chen and J. M. Lin, Anal. Chem., 2011, 83, 8245-8251.

16 J. Jiang, Y. He, S. Y. Li and H. Cui, Chem. Commun., 2012, 48, 9634-9636.

17 X. F. Jia, J. Li and E. K. Wang, Nanoscale, 2012, 4, 5572-5575.

18 H. Z. Zheng, Q. L. Wang, Y. J. Long, H. J. Zhang, X. X. Huang and R. Zhu, Chem. Commun., 2011, 47, 10650-10652.

19 L. Zhou, Y. H. Lin, Z. Z. Huang, J. S. Ren and X. G. Qu, Chem. Commun., 2012, 48, 1147-1149.

20 S. N. Baker and G. A. Baker, Angew. Chem., Int. Ed., 2010, 49, 6726-6744.

21 W. S. Hummers and R. E. Offeman, J. Am. Chem. Soc., 1958, 80, 1339.

22 X. B. Fan, W. C. Peng, Y. Li, X. Y. Li, S. L. Wang, G. L. Zhang and F. B. Zhang, Adv. Mater., 2008, 20, 4490-4493.

23 A. B. Sproul and M. A. Green, J. Appl. Phys., 1991, 70, 846854. 